\title{
Influence of Preoperative Physical Function on Gait 1 Month After Total Knee Arthroplasty
}

\author{
So Young Lee ${ }^{1}$, Bo Ryun Kim², Sang Rim Kim², Eun Young Han ${ }^{1}$, Kwang Woo Nam², Yong-Geun Park ${ }^{2}$ \\ Departments of ${ }^{1}$ Rehabilitation Medicine and ${ }^{2}$ Orthopedic Surgery, Regional Rheumatoid and Degenerative Arthritis Center, Jeju National \\ University Hospital, Jeju National University School of Medicine, Jeju, Korea
}

Corresponding Author: Bo Ryun Kim, MD, PhD Department of Rehabilitation Medicine, Regional Rheumatoid and Degenerative Arthritis Center, Jeju National University Hospital, Jeju National University School of Medicine, 15 Aran 13-gil, Jeju 63241, Korea

Tel: $+82-64-717-1672$

Fax: +82-64-717-1131

E-mail: brkim08@gmail.com

Received: October 22, 2017 Revised: December 4, 2017 Accepted: December 8, 2017
Background: This study aimed to identify the preoperative physical performance factors that predict gait speed and endurance 1 month after total knee arthroplasty (TKA). Methods: This prospective cohort study included all patients who underwent unilateral primary TKA in December 2014-2016. Before and at 1 month after TKA, gait variables, bilateral isometric knee extensor and flexor strength, and range of motion (ROM) (flexion and extension) of the operated knee were measured; further, a 6-minute walk test (6MWT), a Timed Up and Go (TUG) test, and a stair-climbing test (SCT) were conducted. The Western Ontario and McMaster Universities Osteoarthritis Index (WOMAC), the EuroOoL 5-dimension (EQ-5D) questionnaire, and the visual analogue scale (VAS) of knee pain were also completed. Results: A total of 84 patients were included. Bivariate analyses showed that postoperative gait speed significantly and positively correlated with preoperative gait speed, gait cadence, ROM of knee flexion, and 6MWT, but significantly and negatively correlated with age and preoperative TUG test, SCT-descent, WOMAC-Function score, and VAS score. The postoperative 6MWT significantly and positively correlated with preoperative gait cadence and 6MWT, but significantly and negatively with preoperative body mass index (BMI), TUG test, SCT-ascent, and WOMAC-Function score. Linear regression analyses showed that age, preoperative ROM of knee flexion, and TUG test were independent predictors of postoperative gait speed, while preoperative BMI and 6MWT predicted postoperative 6MWT. Conclusion: These preoperative predictive factors will be useful in developing pre- and postoperative rehabilitation strategies aimed at improving gait function in the early stages after TKA.

Key Words: Osteoarthritis, Knee, Arthroplasty, Gait, Rehabilitation

\section{INTRODUCTION}

Knee osteoarthritis (OA) is a very common disease and a leading cause of joint pain and swelling, functional disability, and impaired quality of life ${ }^{1)}$. Total knee arthroplasty (TKA) is the most common and effective surgical intervention for end-stage knee $\mathrm{OA}^{2)}$. Many studies show that compared to the preoperative situation, TKA significantly improves knee pain, self-reported physical function, and quality of life ${ }^{3-5)}$.

However, despite successful TKA, multiple performancebased physical functions, including gait function, remain impaired $^{6-8)}$. In particular, while the gait speed of patients improves slowly in the months after surgery, gait speed limitations continue to persist after the first year. Furthermore, Bade et al. ${ }^{9)}$ showed that compared to healthy adults, patients still have deficits in the Timed Up and Go (TUG) test and the 6-minute walk test (6MWT) 6 months after TKA. Moreover,
Walsh et al. ${ }^{10)}$ reported that compared to an age-matched group of healthy individuals, patients were consistently having difficulties to ascend and descend stairs after TKA. Therefore, recovery of gait function after TKA is a major concern and is considered the most important factor when predicting the physical function of a patient after TKA. However, studies regarding preoperative factors predictive of postoperative gait speed and gait endurance simultaneously following TKA are limited.

The identification of preoperative predictors of gait function in the first month following TKA is of great importance. Since the first month after TKA is the period wherein a patient is generally returning home after surgery, these predictors will be helpful in determining their expected level of independence in community-based activities and predicting the need for postoperative locomotor rehabilitation and home support $^{11)}$.

Therefore, this study was conducted to identify preopera- 
tive physical performance factors that predict postoperative gait speed and endurance. The implications of the study findings for pre- and postoperative rehabilitation strategies that aim to improve gait function in patients scheduled for TKA are also discussed.

\section{MATERIALS AND METHODS}

\section{Participants}

All patients who were diagnosed with end-stage primary $\mathrm{OA}$ of the knee, who were scheduled to undergo unilateral primary TKA in December 2014-2016 at the Department of Orthopedic Surgery in Jeju National University Hospital, and who consented to participate in the study were recruited prospectively. Two weeks after surgery, all patients were transferred to the rehabilitation department of the hospital. Patients were included in the study if they could walk independently with or without an ambulatory aid before surgery. Patients who had previous neurological or orthopedic disease that caused ambulatory deficits (e.g., fracture or unstable cardio-respiratory disease) were excluded.

All participants were informed about the nature of the study and the risks and benefits, and all provided written consent to participate. The study protocol was approved by the Institutional Review Board of Jeju National University Hospital (JEJUNUH 2017-11-007).

\section{Rehabilitation Protocol}

After surgery, all patients underwent a rehabilitation program that included a passive knee range of motion (ROM) exercise, progressive resistance strengthening exercises, gait training, aerobic exercise using an ergometer, functional training for transfers and stair climbing, and physical modalities such as cryotherapy and transcutaneous electrical nerve stimulation to relieve the knee pain and swelling. The progressive resistance strengthening exercises consisted of concentric knee extensions and flexions and hip abduction and adduction exercises that were performed on air-resistance machines (HUR Co., Kokkola, Finland) at 30\% of the patient's one-repetition maximum (1 RM) for 3 sets of 15 repetitions in 30-minute sessions. The 1 RM was reassessed every week, and the training prescription was determined and increased to a new resistance level until the patients could perform $60 \%$ of their $1 \mathrm{RM}$ for three sets of ten repetitions. All rehabilitation programs were performed 5 times per week for 2 weeks after surgery under the supervision of physical therapists.

\section{Outcome Measures}

All patients completed a series of assessments before and at 1 month after surgery. The physical performance of all patients was determined by conducting instrumental gait analysis of spatiotemporal variables, measuring the isometric knee extensor and flexor strength of the surgical and nonsurgical knees and the ROM of flexion and extension of the surgical knee, and conducting a 6MWT, a TUG test, and a stair-climbing test (SCT). Self-reported physical function was evaluated using the Western Ontario and McMaster Universities Osteoarthritis Index (WOMAC), while the self-reported quality of life was evaluated using the EuroQoL five-dimension (EO-5D) questionnaire. A visual analogue scale (VAS) of knee pain was also recorded ${ }^{12)}$.

\section{1) Assessment of performance-based physical function}

\section{(1) Gait analysis}

The spatiotemporal variables of gait were measured using a validated wireless inertial sensing device (G-Walk, BTS Bioengineering S.p.A., Milan, Italy). Each patient was instructed to wear the semi-elastic back-belt device on the waist, to measure the acceleration values of the 3 anatomical axes (anteroposterior, mediolateral, and vertical). Consequently, patients were instructed to stand and remain in an upright posture for a few seconds and then walk barefoot along an 8-m pathway at a comfortable speed as naturally as possible. Gait data were collected and transmitted via Bluetooth to a personal computer, where the data were processed using the BTS G-Walk system, which is a dedicated software that measures gait variables such as gait speed, cadence, stride length, duration of gait cycle, stance phase, swing phase, and double and single supports. From the typical gait curve with 2 positive and one negative peak, the relevant timing was calculated; the second positive peak was considered as the instant of foot contact on the ground. Therefore, the distance between the two successive foot contacts on the ground was considered as the step length, while the distance between three successive foot contacts was considered as the stride length. The first and last steps of the 8- $\mathrm{m}$ walk were removed from all calculations considering the abnormal patterns at the initiation of gait or the psychological responses at the termination of gait $^{13)}$.

\section{(2) Isometric strength measurement of the knee extensors and flexors}

The maximal isometric strength of the bilateral knee extensors and flexors was measured using an isokinetic dynamometer (HUMAC Norm, CSMI, Stoughton, MA, USA). All patients relaxed their muscles with light stretching before the test. The participants were then seated on a specially designed chair so that they attained a hip angle of approximately $85^{\circ 14}$. The distal shin pad of the dynamometer was attached $2-3 \mathrm{~cm}$ proximal to the lateral malleolus using a 
strap. Additional straps were applied across the chest, pelvis, and midthigh to minimize inappropriate trunk movements during thigh muscle contractions. The alignment between the dynamometer rotational axis and the knee joint rotational axis (lateral femoral epicondyle) was adjusted at the beginning of each trial. The gravity effect torque was recorded for each subject and used to correct the torque measurements during all tests. Patients were instructed to grasp the sidebars during the testing procedure. The length of the arm measured from the lateral femoral epicondyle to the center of the force transducer at the shin was kept constant. Data were obtained from digitized signals. After a structured warm-up with the knee joint fixed at $60^{\circ}$ of flexion for maximal isometric force generation ${ }^{15)}$, the patients were instructed to perform maximal voluntary contractions until the torque did not increase by more than $5 \%$ during three successive attempts. Knee flexion and extension were performed as discrete movements in a single direction (i.e., nonreciprocal). Each contraction lasted 4-5 seconds, with 2-min rest intervals. During each attempt, the physical therapist loudly encouraged the patients to achieve their maximal performance. After a 5-min rest, the same procedure was performed with the other lower limb.

The variables that were analyzed included extensor and flexor peak torque, the ratio of hamstring to quadriceps strength ( $\mathrm{H} / \mathrm{Q}$ ratio), and the difference in strength of the extensors and flexors between the surgical and nonsurgical knees expressed as a percentage of the nonsurgical knee performance.

\section{(3) ROM measurement of the knee}

Available knee ROM was measured using a standard longarm goniometer. The axis of the goniometer was aligned with the center of the lateral epicondyle of the femur. The distal arm of the goniometer was aligned with the lateral malleolus, and the proximal arm was aligned with the greater trochanter of the femur. Knee flexion ROM was determined as the value of maximal active bending of the knee while the patient was lying in the supine position. Knee extension ROM was determined as the angle of maximal active straightening while the patient's heel was propped on a $10-\mathrm{cm}$ wooden block. If a patient achieved hyperextension during knee ROM assessment, then the degrees of extension beyond zero were recorded as a negative value ${ }^{16)}$.

\section{(4) 6-minute walk test (6MWT)}

The 6MWT is a performance-based measurement of functional walking capacity and gait endurance in adults. Patients were instructed to walk as far as possible for 6 min along a $50-m$ hallway that was marked with lines ${ }^{177}$.

(5) Timed Up and Go test (TUG test)

The TUG test evaluates dynamic balance performance. Each patient sat with their back against a chair (seat height,
$44 \mathrm{~cm}$; depth, $45 \mathrm{~cm}$; width, $49 \mathrm{~cm}$; and arm rest height, 64 $\mathrm{cm})$ that was placed at the end of a marked 3-m distance. Patients were instructed to stand up on the word "go," walk at a comfortable speed past the 3-m mark, turn around, walk back, and sit down again in the chair without physical assistance, all the while being timed ${ }^{18)}$.

\section{(6) Stair climbing test (SCT)}

The SCT was measured as the time required to ascend and descend a flight of stairs (12 steps, each $17 \mathrm{~cm}$ high and $25 \mathrm{~cm}$ wide). Patients were instructed to ascend or descend the stairs as fast as possible on the word "go." Each trial was performed with a 5-min rest interval and the best score was recorded ${ }^{19)}$.

\section{2) Assessment of self-reported physical function, pain, and quality of life}

\section{(1) Western Ontario McMaster Universities Osteoarthritis Index (WOMAC)}

The multidimensional WOMAC questionnaire includes questions about pain, stiffness, and physical function. It has been used previously to measure self-reported disability in patients with knee OA. The questionnaires include 5 pain, 2 stiffness, and 17 physical function variables. Each of these variables is scored using the Likert scale $(0$, none; 1 , slight; 2 , moderate; 3 , very; and 4, extremely), which is employed to measure the outcomes in rheumatology clinical trials. Thus, the Likert scale is used to determine the degree of pain, stiffness, and difficulty experienced in performing each of 17 activities in the preceding 48 hours. Higher scores indicate greater levels of pain, stiffness, and difficulty ${ }^{20}$. The scores for pain, stiffness, and physical function variables are summed up to yield the WOMAC-Pain (range, 0-20), WOMAC-Stiffness (range, $0-8$ ), and WOMAC-Function (range, 0-68) subscores.

\section{(2) EuroQoL five (EQ-5D) dimension questionnaires}

The EQ-5D questionnaire is used to evaluate self-reported quality of life. The EQ-5D index is widely used to measure the general health status. The instrument consists of questionnaires with 5 dimensions: mobility, self-care, usual activities, pain/discomfort, and anxiety/depression. Each dimension is represented by one question that has 3 severity levels (no problems, some or moderate problems, and extreme problems). The scores were transformed using utility weights derived from the general Korean population and ranged from -1 to 1 . Higher scores indicate better overall health status. The formula for the EQ-5D index has been presented by Kim et al. ${ }^{211}$.

(3) Visual analogue scale

Patients were instructed to evaluate their level of knee pain using a VAS. The scale consisted of a $10-\mathrm{cm}^{-l o n g}$ horizon- 
tal line that ranged from completely satisfied to totally unsatisfied. Facial expressions were placed above the line to express satisfaction visually. The satisfaction VAS system ranged from 0 (worst, totally unsatisfied) to 10 (best, completely satisfied) points ${ }^{22}$. All patients were instructed to mark the line on the scale at the point that matched their satisfaction.

\section{Statistical Analysis}

All statistical analyses were performed using IBM SPSS Statistics ver. 20.0 (IBM Co., Armonk, NY, USA). All variables were subjected to descriptive statistics. Pearson correlation analysis was used to assess the relationships between preoperative factors and postoperative gait speed and endurance. Multivariate regression analysis using a backward selection linear regression model was employed to determine which preoperative factors best explained gait speed and endurance 1 month after TKA. A p-value of $<0.05$ was considered to indicate statistical significance.

\section{RESULTS}

Eighty-four patients were recruited: 76 female and 8 male patients. Table 1 presents their baseline demographic and disease-related characteristics. The average age and body mass index (BMI) were $72.0 \pm 6.0$ years and $27.1 \pm 3.6 \mathrm{~kg} / \mathrm{m}^{2}$, respectively. Among the 84 patients, 63 (75.0\%) had KellgrenLawrence grade $4 \mathrm{OA}$.

\section{Preoperative and postoperative evaluation of per- formance-based physical function, self-reported physical function, and quality of life}

Table 2 presents the average preoperative and postoperative physical test performances, physical functions, and quality of life scores of the patients.

\section{Correlation between the preoperative factors and postoperative gait function}

Bivariate analyses showed that postoperative gait speed was significantly and positively correlated with the preoperative gait speed $(r=0.36, p=0.001)$, gait cadence $(r=0.27$, $p=0.010), R O M$ of knee flexion $(r=0.27, p=0.010)$, and 6MWT $(r=0.31, p=0.010)$, but significantly and negatively correlated with age $(r=-0.33, p<0.010)$, preoperative TUG test $(r=-0.40$, $p<0.001)$, SCT-descent $(r=-0.38, p<0.001)$, WOMAC-Function score $(r=-0.35, p=0.010)$, and VAS score $(r=-0.29, p=0.010)$. Postoperative 6MWT was significantly and positively correlated with preoperative gait cadence $(r=0.25, p=0.020)$ and the 6MWT $(r=0.51, p<0.001)$, but significantly and negatively correlated with the preoperative BMI $(r=-0.23, p=0.030)$, TUG test $(r=-0.38, p<0.001)$, SCT-ascent $(r=-0.38, p<0.001)$, and WOMAC-Function score $(r=-0.30, p=0.010)$ (Table 3).

\section{Preoperative factors predicting the postoperative gait function by multivariate linear regression analysis}

Linear regression analyses identified age $(\beta=-0.24, p=0.010)$, preoperative ROM of knee flexion $(\beta=0.23, p=0.020)$, and preoperative TUG test $(\beta=-0.24, p=0.020)$ as predictors of postoperative gait speed $\left(R^{2}=0.31\right)$, whereas preoperative BMI $(\beta=-0.22, p=0.020)$ and 6MWT $(\beta=0.41, p<0.001)$ predicted postoperative gait endurance $\left(R^{2}=0.31\right)$ (Table 4 ).

\section{DISCUSSION}

We identified the preoperative physical performance factors that predicted gait speed and endurance 1 month after TKA. The study showed that in the first month after TKA, age, preoperative ROM of surgical knee flexion, and balance were the best independent predictors of postoperative gait speed, while preoperative BMl and gait endurance were the best independent predictors of postoperative gait endurance.

Among the various outcome measures of TKA, gait speed is a particularly useful variable for assessing the functional capacity. Gait speed can be used not only as a simple instrument for monitoring postoperative recovery but also can

Table 1. Demographic and disease-related characteristics of the subjects $(n=84)$

\begin{tabular}{lc}
\hline \hline Variable & Value \\
\hline Age $(\mathrm{yr})$ & $72.0 \pm 6.0$ \\
Sex, male:female & \\
$\quad$ Male & $7(9.5)$ \\
Female & $76(90.5)$ \\
\hline Height $(\mathrm{cm})$ & $152.7 \pm 6.1$ \\
Weight $(\mathrm{kg})$ & $63.2 \pm 9.2$ \\
\hline Body mass index $\left(\mathrm{kg} / \mathrm{m}^{2}\right)$ & $27.1 \pm 3.6$ \\
K-L grades & \\
\hline Grade 3 & $21(25.0)$ \\
\hline Grade 4 & $63(75.0)$ \\
\hline Lesion side & \\
Right & $60(71.4)$ \\
Left & $24(28.6)$ \\
Comorbidities & \\
\hline Diabetes mellitus & $16(19.0)$ \\
\hline Hypertension & $65(77.4)$ \\
Spine disease & $9(10.7)$ \\
Osteoporosis & $42(50.0)$ \\
\hline
\end{tabular}

Values are presented as mean \pm standard deviation or number of cases (\%).

K-L, Kellgren-Lawrence. 
serve as a screening tool for comorbidity ${ }^{23-25)}$.

As expected, among the variables we measured, age was strongly associated with, and the most powerful predictor of, postoperative gait speed. A recent study ${ }^{26)}$ also found that the association between age and fast gait speed over time after TKA was negative and nonlinear. In addition, Cooper et al. ${ }^{27)}$ showed that age is the strongest predictor of gait speed at 6 weeks and 6 months after TKA. This association is explained by the fact that older patients have a worse perceived function and physical performance when walking after TKA.
Preoperative ROM of surgical knee flexion was also a predictor of postoperative gait speed. By contrast, Brown et al. $^{28)}$ showed that while preoperative flexion ROM of the surgical knee correlated with functional tasks, including the 6MWT and descending a second flight of stairs, it did not correlate with gait speed. However, consistent with our findings, Pua et al. ${ }^{26)}$ found that postoperative knee flexion ROM has a significant clinical influence on gait speed after TKA and is linearly correlated. Notably, walking is usually performed with the knees flexed at a maximum of $67^{\circ}{ }^{.29)}$ however, our patients had a mean preoperative knee flexion ROM

Table 2. Preoperative and postoperative evaluation of physical performance, physical function, and quality of life

\begin{tabular}{|c|c|c|}
\hline Variable & Preoperative & Postoperative \\
\hline \multicolumn{3}{|l|}{ Gait linear parameters } \\
\hline Gait speed (m/sec) & $0.9 \pm 0.2$ & $1.0 \pm 0.1$ \\
\hline Cadence (steps/min) & $103.3 \pm 13.9$ & $113.6 \pm 10.6$ \\
\hline Stride length $(\mathrm{cm})$ & $101.1 \pm 11.1$ & $108.8 \pm 12.8$ \\
\hline Gait cycle duration (sec) & $1.2 \pm 0.2$ & $1.2 \pm 1.1$ \\
\hline Stance phase duration ( $\%$ of gait cycle) & $65.2 \pm 2.4$ & $64.7 \pm 1.6$ \\
\hline Swing phase duration ( $\%$ of gait cycle) & $34.8 \pm 2.4$ & $35.3 \pm 1.6$ \\
\hline Double support duration (\% of gait cycle) & $30.3 \pm 5.3$ & $29.6 \pm 3.3$ \\
\hline Single support duration (\% of gait cycle) & $34.7 \pm 2.5$ & $35.1 \pm 1.6$ \\
\hline \multicolumn{3}{|l|}{ Isometric strength test } \\
\hline PT extensor of surgical knee $(\mathrm{N} / \mathrm{m} / \mathrm{kg} \mathrm{BW})$ & $76.5 \pm 26.7$ & $50.2 \pm 20.2$ \\
\hline PT flexor of surgical knee (N/m/kg BW) & $48.8 \pm 13.3$ & $48.7 \pm 14.9$ \\
\hline $\mathrm{H} / \mathrm{Q}$ ratio of surgical knee & $0.7 \pm 0.3$ & $1.1 \pm 0.6$ \\
\hline PT extensor of nonsurgical knee $(\mathrm{N} / \mathrm{m} / \mathrm{kg}$ BW) & $95.5 \pm 31.9$ & $100.7 \pm 32.5$ \\
\hline PT flexor of nonsurgical knee (N/m/kg BW) & $51.6 \pm 16.5$ & $55.9 \pm 17.6$ \\
\hline $\mathrm{H} / \mathrm{Q}$ ratio of nonsurgical knee & $0.6 \pm 0.2$ & $0.6 \pm 0.2$ \\
\hline Deficit of extensor $(\%)$ & $20.5 \pm 20.4$ & $48.1 \pm 20.3$ \\
\hline Deficit of flexor (\%) & $5.2 \pm 15.4$ & $13.1 \pm 18.0$ \\
\hline \multicolumn{3}{|l|}{ ROM (degree) } \\
\hline Knee flexion & $129.5 \pm 9.8$ & $114.9 \pm 11.0$ \\
\hline Knee extension & $-7.9 \pm 7.2$ & $-4.3 \pm 5.4$ \\
\hline 6MWT (m) & $314.0 \pm 88.5$ & $363.7 \pm 69.4$ \\
\hline TUG (sec) & $12.0 \pm 2.8$ & $10.6 \pm 2.2$ \\
\hline \multicolumn{3}{|l|}{ SCT (sec) } \\
\hline Ascent & $15.1 \pm 6.1$ & $16.0 \pm 4.4$ \\
\hline Descent & $17.4 \pm 7.0$ & $17.3 \pm 5.2$ \\
\hline \multicolumn{3}{|l|}{ WOMAC } \\
\hline Pain & $8.5 \pm 3.0$ & $5.2 \pm 2.4$ \\
\hline Stiffness & $2.5 \pm 1.5$ & $1.9 \pm 1.0$ \\
\hline Function & $26.8 \pm 8.2$ & $21.1 \pm 6.4$ \\
\hline EQ-5D & $0.6 \pm 0.1$ & $0.7 \pm 0.1$ \\
\hline VAS & $6.8 \pm 1.6$ & $3.7 \pm 1.4$ \\
\hline
\end{tabular}

Values are presented as mean \pm standard deviation.

PT, peak torque; BW, body weight; H/Q, hamstring to quadriceps strength; ROM, range of motion; 6MWT, 6-minute walk test; TUG, Timed Up and Go; SCT, stair climbing test; WOMAC, Western Ontario McMaster Universities Osteoarthritis Index; EQ-5D, EuroQoL 5 dimensions; VAS, visual analogue scale. 
of $129^{\circ}$. While the correlation between preoperative ROM of surgical knee flexion and postoperative gait speed remains unclear, it may relate to greater preoperative participation in daily and physical activities that demand high knee flexion $^{30)}$. Further investigation of this pathophysiology is warranted. Our results support calls to address the ROM of the surgical knee and to introduce prerehabilitation strategies that focus on stretching exercises of the knee joint that will improve gait.

Mizner et al. ${ }^{31)}$ reported that preoperative quadriceps strength strongly predicts functional variables 1 year after TKA. Valtonen et al. ${ }^{32)}$ also found that knee flexor power was independently associated with gait speed in patients with knee OA. However, our results showed that preoperative quadriceps and hamstring strength did not significantly affect gait speed 1 month after TKA. By contrast, the preoperative

Table 3. Correlation between preoperative factors and postoperative gait function

\begin{tabular}{|c|c|c|}
\hline \multirow{2}{*}{ Variable } & \multicolumn{2}{|c|}{ Correlation coefficients (r) } \\
\hline & Postoperative gait speed & Postoperative gait endurance \\
\hline Age & $-0.33^{\dagger}$ & -0.10 \\
\hline Body mass index & -0.13 & $-0.23 *$ \\
\hline \multicolumn{3}{|l|}{ Gait linear parameters } \\
\hline Gait speed $(\mathrm{m} / \mathrm{sec})$ & $0.36^{\dagger}$ & 0.19 \\
\hline Cadence (steps/min) & $0.27 *$ & $0.25 *$ \\
\hline Stride length $(\mathrm{cm})$ & 0.31 & 0.07 \\
\hline Gait cycle duration (sec) & -0.24 & -0.18 \\
\hline Stance phase duration ( $\%$ of gait cycle) & 0.04 & 0.09 \\
\hline Swing phase duration (\% of gait cycle) & -0.04 & -0.09 \\
\hline Double support duration ( $\%$ of gait cycle) & 0.03 & 0.06 \\
\hline Single support duration ( $\%$ of gait cycle) & -0.04 & -0.06 \\
\hline \multicolumn{3}{|l|}{ Isometric strength test } \\
\hline PT extensor of surgical knee $(\mathrm{N} / \mathrm{m} / \mathrm{kg}$ BW) & 0.18 & 0.09 \\
\hline PT flexor of surgical knee (N/m/kg BW) & 0.20 & 0.02 \\
\hline $\mathrm{H} / \mathrm{Q}$ ratio of surgical knee & 0.03 & -0.06 \\
\hline PT extensor of nonsurgical knee $(\mathrm{N} / \mathrm{m} / \mathrm{kg} \mathrm{BW})$ & 0.21 & 0.05 \\
\hline PT flexor of nonsurgical knee (N/m/kg BW) & 0.12 & 0.01 \\
\hline $\mathrm{H} / \mathrm{Q}$ ratio of nonsurgical knee & -0.12 & -0.09 \\
\hline Deficit of extensor (\%) & 0.08 & 0.06 \\
\hline Deficit of flexor $(\%)$ & 0.00 & 0.09 \\
\hline \multicolumn{3}{|l|}{ ROM (degree) } \\
\hline Knee flexion & $0.27 *$ & 0.004 \\
\hline Knee extension & 0.19 & -0.03 \\
\hline 6MWT (m) & $0.31^{*}$ & $0.51^{\dagger}$ \\
\hline TUG (sec) & $-0.40^{\dagger}$ & $-0.38^{\dagger}$ \\
\hline \multicolumn{3}{|l|}{ SCT (sec) } \\
\hline Ascent & -0.15 & $-0.37^{\dagger}$ \\
\hline Descent & $-0.38^{\dagger}$ & -0.38 \\
\hline \multicolumn{3}{|l|}{ WOMAC } \\
\hline Pain & -0.16 & -0.11 \\
\hline Stiffness & -0.07 & -0.16 \\
\hline Function & $-0.35^{\dagger}$ & $-0.30^{\dagger}$ \\
\hline EQ-5D & 0.11 & 0.05 \\
\hline VAS & $-0.29^{\dagger}$ & -0.05 \\
\hline
\end{tabular}

PT, peak torque; BW, body weight; H/Q, hamstring to quadriceps strength; ROM, range of motion; 6MWT, 6-minute walk test; TUG, Timed Up and Go; SCT, stair climbing test; WOMAC, Western Ontario McMaster Universities Osteoarthritis Index; EQ-5D, EuroQoL 5 dimensions; VAS, visual analog scale.

" $\mathrm{p}<0.05 .{ }^{\dagger} \mathrm{p}<0.01$. 
Table 4. Preoperative factors predicting the postoperative gait function by multivariate linear regression analysis

\begin{tabular}{lrrr}
\hline \hline \multicolumn{3}{l}{ Outcome/independent predictor Standardized $\beta$} & p-value Adjusted $\mathrm{R}^{2}$ \\
\hline Gait speed & & & \\
Age & -0.24 & 0.010 & 0.31 \\
ROM of surgical knee flexion & 0.23 & 0.020 & \\
TUG & -0.24 & 0.020 & \\
Gait endurance & & & \\
BMI & -0.22 & 0.020 & 0.31 \\
6MWT & 0.41 & $<0.001$ & \\
\hline
\end{tabular}

ROM, range of motion; TUG, Timed Up and Go; BMI, body mass index; 6MWT, 6-minute walk test.

TUG test was an independent predictor of postoperative gait speed in our study. The TUG test has acceptable concurrent validity for measuring dynamic balance and high repeatability in patients awaiting TKA ${ }^{18)}$. Similarly, Ko et al. ${ }^{33)}$ found that TUG test predicted physical activity and WOMACFunction scores. The association between TUG test and postoperative gait speed may be due to their sit-to-stand element; TUG test performance depends on a combination of neuromuscular components (walking ability, lower limb strength, and balance) that also play important roles in gait speed.

Along with gait speed, the 6MWT is commonly used to evaluate ambulatory capacity because it is a simple and clinically relevant measure of exercise endurance. The 6MWT has been shown as a robust measurement of functional mobility during the long-term recovery of patients after TKA ${ }^{33)}$ and is a highly reliable measurement tool in patients who underwent primary TKA ${ }^{34)}$. Here, we found that preoperative BMI was the most powerful predictor of postoperative gait endurance in the first month following TKA. These results are consistent with and support other studies that show walking distance on the $6 \mathrm{MWT}$ is negatively correlated with $\mathrm{BMI}^{35,36)}$. Cooper et al. $^{27)}$ also showed that BMl predicts daily activities 6 weeks and 6 months after TKA. The association between poor 6MWT values and obesity may be due to the fact that obesity is associated with worse perceived function and higher rates of complication after TKA. In addition, overweight individuals are less physically active and have a lower capacity for activity, which limits their improvement after TKA. These results suggest that weight reduction before surgery may improve postoperative gait function.

The preoperative 6MWT also predicted gait endurance in the first month after TKA. This finding is supported by Parent and Moffet ${ }^{37)}$, who showed that the preoperative $6 \mathrm{MWT}$, knee pain, and knee flexion predict locomotor ability 2 months after TKA with good accuracy. This association probably reflects the fact that the baseline value of a given measure is often a strong predictor of the same measure later in time ${ }^{38,39)}$.
This study has several limitations. First, the study results may not be generalized to all TKA surgeries because this study only included people with OA who underwent a unilateral primary TKA. Therefore, similar studies on patients after a revision or bilateral TKA or patients undergoing a first TKA for a diagnosis other than OA are warranted. In addition, since we did not analyze the postoperative variables at time points other than 1-month post-TKA, we cannot guarantee that the predictors of gait function in our study will also be significant predictors of the performance several years post-TKA. Long-term follow-up studies that address this are also warranted. Second, we did not compare our patients to those who did not undergo postoperative rehabilitation. Further studies on the causal relationships between the postoperative gait variables and the effects of our intensive rehabilitation program are needed.

In conclusion, this study showed that age, preoperative ROM of knee flexion, and balance ability significantly influenced postoperative gait speed. In addition, preoperative $\mathrm{BMI}$ and gait endurance influenced postoperative gait endurance. This study suggests that variable preoperative rehabilitation strategies that focus on improving knee ROM, balance, and endurance training will improve gait function after TKA. It also suggests that weight reduction may aid the recovery of gait function in the early stages after TKA. Furthermore, we recommend that patients who have a poor prognosis in terms of gait function after TKA should undergo intensive postoperative rehabilitation to improve gait function.

Conflicts of Interest Disclosures: The researchers claim no conflicts of interest.

\section{Acknowledgments}

This work was supported by the research grant of Jeju National University in 2017.

\section{REFERENCES}

1. Peat G, McCarney R, Croft P. Knee pain and osteoarthritis in older adults: a review of community burden and current use of primary health care. Ann Rheum Dis 2001;60:91-7.

2. NIH Consensus Statement on total knee replacement. NIH Consens State Sci Statements 2003;20:1-34.

3. Cushnaghan J, Bennett J, Reading I, Croft P, Byng P, Cox K, et al. Long-term outcome following total knee arthroplasty: a controlled longitudinal study. Ann Rheum Dis 2009;68:642-7.

4. Ethgen $\mathrm{O}$, Bruyère $\mathrm{O}$, Richy $\mathrm{F}$, Dardennes $\mathrm{C}$, Reginster JY. Health-related quality of life in total hip and total knee arthroplasty. A qualitative and systematic review of the literature. J Bone Joint Surg Am 2004;86-A:963-74.

5. Xie F, Lo NN, Pullenayegum EM, Tarride JE, O'Reilly DJ, Goeree R, et al. Evaluation of health outcomes in osteoarthritis 
patients after total knee replacement: a two-year follow-up. Health Qual Life Outcomes 2010;8:87.

6. Meier W, Mizner RL, Marcus RL, Dibble LE, Peters C, Lastayo PC. Total knee arthroplasty: muscle impairments, functional limitations, and recommended rehabilitation approaches. J Orthop Sports Phys Ther 2008;38:246-56.

7. Noble PC, Gordon MJ, Weiss JM, Reddix RN, Conditt MA, Mathis KB. Does total knee replacement restore normal knee function? Clin Orthop Relat Res 2005;(431):157-65.

8. Milner CE. Is gait normal after total knee arthroplasty? Systematic review of the literature. J Orthop Sci 2009;14:114-20.

9. Bade MJ, Kohrt WM, Stevens-Lapsley JE. Outcomes before and after total knee arthroplasty compared to healthy adults. J Orthop Sports Phys Ther 2010;40:559-67.

10. Walsh M, Woodhouse LJ, Thomas SG, Finch E. Physical impairments and functional limitations: a comparison of individuals 1 year after total knee arthroplasty with control subjects. Phys Ther 1998;78:248-58.

11. Christiansen CL, Bade MJ, Weitzenkamp DA, Stevens-Lapsley JE. Factors predicting weight-bearing asymmetry 1month after unilateral total knee arthroplasty: a cross-sectional study. Gait Posture 2013;37:363-7.

12. Hyun CW, Kim BR, Han EY, Kim SR. Preoperative physical performance predictors of self-reported physical function and quality of life in patients scheduled for total knee arthroplasty. J Phys Ther Sci 2016;28:3220-6.

13. Bugané F, Benedetti MG, Casadio G, Attala S, Biagi F, Manca $\mathrm{M}$, et al. Estimation of spatial-temporal gait parameters in level walking based on a single accelerometer: validation on normal subjects by standard gait analysis. Comput Methods Programs Biomed 2012;108:129-37.

14. Maffiuletti NA, Bizzini M, Desbrosses K, Babault N, Munzinger U. Reliability of knee extension and flexion measurements using the Con-Trex isokinetic dynamometer. Clin Physiol Funct Imaging 2007;27:346-53.

15. Thorstensson A, Karlsson J. Fatiguability and fibre composition of human skeletal muscle. Acta Physiol Scand 1976;98:318-22.

16. Cibere J, Bellamy N, Thorne A, Esdaile JM, McGorm KJ, Chalmers A, et al. Reliability of the knee examination in osteoarthritis: effect of standardization. Arthritis Rheum 2004; 50:458-68.

17. Enright PL, McBurnie MA, Bittner V, Tracy RP, McNamara R, Arnold A, et al. The 6-min walk test: a quick measure of functional status in elderly adults. Chest 2003;123:387-98.

18. Podsiadlo D, Richardson S. The timed "Up \& Go": a test of basic functional mobility for frail elderly persons. J Am Geriatr Soc 1991;39:142-8.

19. Almeida GJ, Schroeder CA, Gil AB, Fitzgerald GK, Piva SR. Interrater reliability and validity of the stair ascend/descend test in subjects with total knee arthroplasty. Arch Phys Med Rehabil 2010;91:932-8.

20. Bellamy N, Buchanan WW, Goldsmith $\mathrm{CH}$, Campbell J, Stitt LW. Validation study of WOMAC: a health status instrument for measuring clinically important patient relevant outcomes to antirheumatic drug therapy in patients with osteoarthritis of the hip or knee. J Rheumatol 1988;15:1833-40.

21. Kim MH, Cho YS, Uhm WS, Kim S, Bae SC. Cross-cultural adaptation and validation of the Korean version of the EQ-5D in patients with rheumatic diseases. Qual Life Res 2005;14:1401-6.

22. Carlsson AM. Assessment of chronic pain. I. Aspects of the reliability and validity of the visual analogue scale. Pain 1983;16:87101.

23. Abbasi-Bafghi H, Fallah-Yakhdani HR, Meijer OG, de Vet HC, Bruijn SM, Yang LY, et al. The effects of knee arthroplasty on walking speed: a meta-analysis. BMC Musculoskelet Disord 2012;13:66.

24. Steffen TM, Hacker TA, Mollinger L. Age- and gender-related test performance in community-dwelling elderly people: SixMinute Walk Test, Berg Balance Scale, Timed Up \& Go Test, and gait speeds. Phys Ther 2002;82:128-37.

25. Lim J, Lim JY. Responsiveness of gait speed to physical exercise interventions in at-risk older adults: a systematic review and meta-analysis. Ann Geriatr Med Res 2017;21:17-23.

26. Pua YH, Seah FJ, Clark RA, Lian-Li Poon C, Tan JW, et al. Factors associated with gait speed recovery after total knee arthroplasty: a longitudinal study. Semin Arthritis Rheum 2017; 46:544-51.

27. Cooper NA, Rakel BA, Zimmerman B, Tonelli SM, Herr KA, Clark CR, et al. Predictors of multidimensional functional outcomes after total knee arthroplasty. J Orthop Res 2017 May 4 [Epub]. https://doi.org/10.1002/jor.23596.

28. Brown K, Kachelman J, Topp R, Quesada PM, Nyland J, Malkani A, et al. Predictors of functional task performance among patients scheduled for total knee arthroplasty. J Strength Cond Res 2009;23:436-43.

29. Rowe PJ, Myles CM, Walker C, Nutton R. Knee joint kinematics in gait and other functional activities measured using flexible electrogoniometry: how much knee motion is sufficient for normal daily life? Gait Posture 2000;12:143-55.

30. Ha CW, Park YB, Song YS, Lee WY, Park YG. Are the current outcome measurement tools appropriate for the evaluation of the knee status in deep flexion range? J Arthroplasty 2016; 31:87-91.

31. Mizner RL, Petterson SC, Stevens JE, Axe MJ, Snyder-Mackler L. Preoperative quadriceps strength predicts functional ability one year after total knee arthroplasty. J Rheumatol 2005;32: 1533-9.

32. Valtonen AM, Pöyhönen T, Manninen M, Heinonen A, Sipilä S. Knee extensor and flexor muscle power explains stair ascension time in patients with unilateral late-stage knee osteoarthritis: a cross-sectional study. Arch Phys Med Rehabil 2015;96: 253-9.

33. Ko V, Naylor JM, Harris IA, Crosbie J, Yeo AE. The six-minute walk test is an excellent predictor of functional ambulation after total knee arthroplasty. BMC Musculoskelet Disord 2013;14: 145.

34. Jakobsen TL, Kehlet H, Bandholm T. Reliability of the 6-min walk test after total knee arthroplasty. Knee Surg Sports Traumatol Arthrosc 2013;21:2625-8.

35. Beriault K, Carpentier AC, Gagnon C, Ménard J, Baillargeon JP, Ardilouze JL, et al. Reproducibility of the 6-minute walk test in obese adults. Int J Sports Med 2009;30:725-7.

36. Larsson UE, Reynisdottir S. The six-minute walk test in outpatients with obesity: reproducibility and known group validity. Physiother Res Int 2008;13:84-93.

37. Parent E, Moffet H. Preoperative predictors of locomotor 
ability two months after total knee arthroplasty for severe osteoarthritis. Arthritis Rheum 2003;49:36-50.

38. Fortin PR, Clarke AE, Joseph L, Liang MH, Tanzer M, Ferland $\mathrm{D}$, et al. Outcomes of total hip and knee replacement: preoperative functional status predicts outcomes at six months after surgery. Arthritis Rheum 1999;42:1722-8.

39. Sharma L, Sinacore J, Daugherty C, Kuesis DT, Stulberg SD, Lewis $\mathrm{M}$, et al. Prognostic factors for functional outcome of total knee replacement: a prospective study. J Gerontol A Biol Sci Med Sci 1996;51:M152-7. 